\title{
Cell surface area regulation in neurons in hippocampal slice cultures is resistant to oxygen-glucose deprivation
}

This article was published in the following Dove Press journal:

Cell Health and Cytoskeleton

24 September 2010

Number of times this article has been viewed

\author{
Natalya Shulyakova ${ }^{1,2}$ \\ Jamie Fong ${ }^{2}$ \\ Diana Diec ${ }^{2}$ \\ Adrian Nahirny ${ }^{1,2}$ \\ Linda R Mills ${ }^{1,2}$ \\ 'Department of Physiology, University \\ of Toronto, Toronto, ON, Canada, \\ M5T 2S8; ${ }^{2}$ Toronto Western Hospital \\ Research Institute, University Health \\ Network, I I-430, 399 Bathurst St, \\ Toronto, ON, Canada, M5T 2S8
}

Background: Neurons swell in response to a variety of insults. The capacity to recover, ie, to shrink, is critical for neuronal function and survival. Studies on dissociated neurons have shown that during swelling and shrinking, neurons reorganize their plasma membrane; as neurons swell, in response to hypo-osmotic media, the bilayer area increases. Upon restoration of normo-osmotic media, neurons shrink, forming transient invaginations of the plasma membrane known as vacuole-like dilations (VLDs), to accommodate the decrease in the bilayer.

Methods: Here we used confocal microscopy to monitor neuronal swelling and shrinking in the three-dimensional (3D) environment of post-natal rat hippocampal slice cultures. To label neurons, we used biolistic transfection, to introduce enhanced green fluorescent protein (eGFP) targeted to the cytoplasm; and a membrane targeted GFP (lckGFP), targeted to the plasma membrane.

Results: Neurons in slice cultures swelled and shrank in response to hypo-osmotic to normoosmotic media changes. Oxygen-glucose deprivation (OGD) caused sustained neuronal swelling; after reperfusion, some neurons recovered but in others, VLD recovery was stalled. OGD did not impair neuronal capacity to recover from a subsequent osmotic challenge.

Conclusion: These results suggest cell surface area regulation (SAR) is an intrinsic property of neurons, and that neuronal capacity for SAR may play an important role in the brain's response to ischemic insults.

Keywords: neurons, swelling, ischemia, cell surface area, hippocampal slice culture

\section{Introduction}

Neurons swell in response to pathophysiological conditions such as seizure, ischemia, metabolic disorders, excitotoxicity, or trauma. The capacity of neurons to recover after swelling, ie, to shrink, is critical for cell function and survival, but little is known about how this process is regulated. While it is recognized that these events involve changes in cell volume, the concomitant changes occurring at the cell membrane are poorly understood. Our studies on dissociated molluscan neurons have established that when neurons swell, and shrink, they reorganize their plasma membrane to accommodate changes in cell surface area. ${ }^{1-5}$ Cell surface area regulation (SAR) is the term we have used to describe this tension-dependent process by which the plasma membrane increases during cell swelling, and decreases during cell shrinking. Our studies have also established that a transient osmotic challenge is a reliable and experimentally convenient method to trigger SAR. In response to a hypotonic challenge, cells swell, recruiting internal membrane stores to the plasma membrane. Upon restoration of normal media, cells rapidly shrink. As they shrink, they form large invaginations of plasma membrane,
Correspondence: Linda R Mills I I-430 Toronto Western Hospital, 399 Bathurst St, Toronto, ON, Canada, M5T 2S8

Tel + I 4166035420

Email linda@uhnres.utoronto.ca or Irmills@gmail.com 
known as vacuole-like dilations (VLDs), at the adherent surface to accommodate the decrease in cell surface area (see Figure 1 and Supplementary Figure 1). Subsequently, the VLDs gradually disappear as active membrane remodeling re-internalizes the excess plasma membrane. In molluscan neurons, the VLD recovery phase requires cortical F-actin dynamics. ${ }^{3,5}$
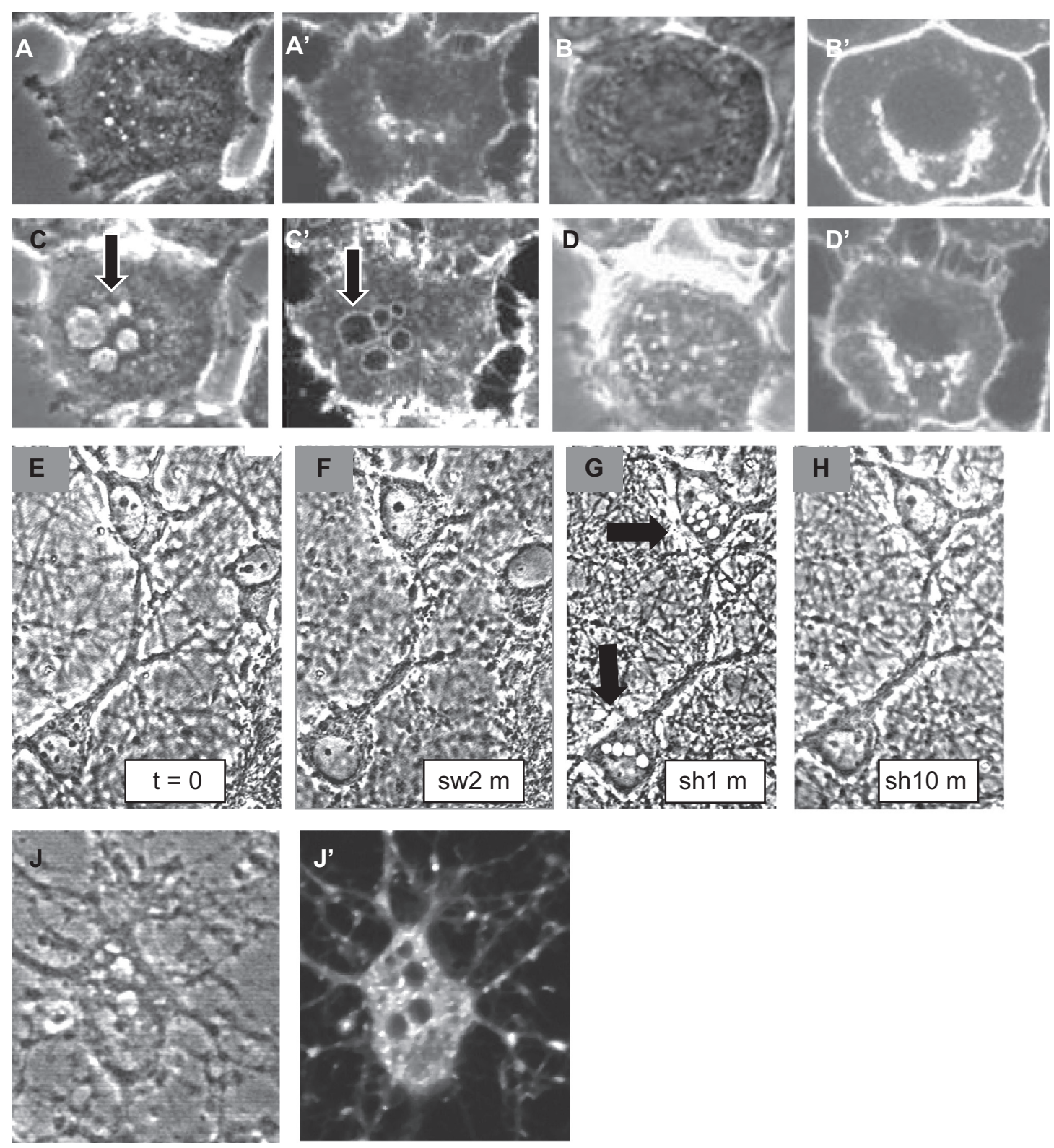

Figure I Visualization of VLD formation and recovery in PCI2 cells expressing IckGFP in the plasma membrane after biolistic transfection. Corresponding pairs of phase contrast and fluorescent images show the cell pre-swell (A and $\mathbf{A}^{\prime}$ ) and during swelling in hyposmotic solution (B and $\mathbf{B}^{\prime}$ ). A return to normo-osmotic medium causes rapid cell shrinking ( $\mathbf{C}$ and $\mathbf{C}^{\prime}$ ) and VLDs (see arrows) form at the adherent surface. Since VLDs are invaginations of the plasma membrane, in cells where only the plasma membrane is labeled, VLD membranes (but not VLD contents) are also labeled.' In the confocal image taken just at the substrate, the VLDs appear as fluorescent rings, as seen elsewhere. ${ }^{1-3}$ In D and D', VLDs spontaneously recover. E-H show high density multilayered hippocampal culture imaged with phase optics during swelling and shrinking. Times shown are minutes in swelling (sw) or shrinking (sh) solutions. E shows pre-swell and in F, somata are swollen and some dendritic beading is evident. In G, as neurons shrink, VLDs form (see arrows). $\mathbf{H}$ shows the status after $10 \mathrm{~min}$. VLD recovery is complete. J and J' show a phase and corresponding fluorescent (confocal) image of a differentiated PCI2 cell during VLD formation. Prior to swelling and shrinking, this cell was loaded with fluo-3, a calcium indicator dye to label the cytoplasm. In this cell, mitochondria are also labeled by mtGFP, a GFP targeted to mitochondria. ${ }^{24}$ Consequently, mitochondria appear as punctuate structures. Since neither the VLD membrane, nor the VLD content, is labeled, as reported elsewhere, ${ }^{1,2}$ in the confocal image, the VLDs appear as dark holes against the background of the fluorescently labeled cytoplasm. 
been established. Indeed, it is difficult to monitor neuronal swelling and shrinking, even in the less complex milieu of a brain slice. Previous studies on brain slices focused on changes in cell volume induced by hypotonicity and ischemia, ${ }^{6-9,20}$ rather than on cell surface area. In the present study, we hypothesized that neurons in the 3D environment of a cultured hippocampal brain slice would demonstrate SAR, and that neuronal capacity for SAR would be impaired by a sublethal ischemic insult, transient oxygen-glucose deprivation (OGD), followed by reperfusion. To monitor swelling and shrinking in neurons in slice cultures, we biolistically transfected enhanced green fluorescent protein (eGFP), targeted to the cytoplasm; or a membrane targeted GFP (lckGFP), targeted to the plasma membrane; and imaged neurons by confocal microscopy.

Our results indicate that neurons in hippocampal slice culture can rapidly swell and shrink in response to single and multiple cycles of an osmotic challenge. Neurons also swelled in response to transient OGD/reperfusion. Both eGFP- and lckGFP-transfected neurons developed VLDs, but VLD recovery appeared to stall, although these same neurons survived for up to two days post-OGD. OGD did not impair neuronal capacity to swell and shrink in response to a subsequent osmotic challenge given within 30-120 min post-reperfusion. These results indicate that neurons in a $3 \mathrm{D}$ environment are capable of SAR, and that neuronal capacity for SAR may be an important determinant of the brain's response to insults that involve cell swelling.

\section{Materials and methods Preparation of organotypic hippocampal slices and dissociated hippocampal cultures}

Hippocampal slice cultures were prepared from seven-day old neonatal Wistar male rats. ${ }^{10,11}$ Fifteen rats from nine separate litters were anesthetized, decapitated, and their brains removed and cooled in dissecting medium (50\% MEM without bicarbonate; $50 \% \mathrm{Ca}^{2+}, \mathrm{Mg}^{2+}$-free Hanks' balanced salt solution; $20 \mathrm{mM}$ HEPES, $6.5 \mathrm{~g} \cdot \mathrm{L}^{-1} \mathrm{D}$-glucose). Coronal slices were prepared using a tissue chopper and transferred to $0.4 \mu \mathrm{m}$ Millicel-CM ${ }^{\circledR}$ culture membranes (Millipore, Billerica, MA), which were transferred to 6-well culture dishes with $1 \mathrm{~mL}$ of culture medium (50\% MEM with Earls salts; $2 \mathrm{mM}$ L-glutamine; 25\% Earle's Balanced Salt Solution (BSS); 25\% horse serum; $5.25 \mathrm{~g} \cdot \mathrm{L}^{-1} \mathrm{D}$-glucose; $20 \mathrm{mM}$ HEPES buffer; and $50 \mathrm{mg} \cdot \mathrm{mL}^{-1}$ streptomycin-penicillin). Cultures were kept at $37^{\circ} \mathrm{C}$ in a humidified incubator with $5 \% \mathrm{CO}_{2}$ and $95 \%$ air, and fed two times a week by $50 \%$ medium exchange. Slices were subject to biolistic transfection at 7 days in vitro (DIV), a time when healthy slices have typically flattened and thinned out. In addition to the slice cultures, some experiments were done using dissociated high density hippocampal cultures and differentiated PC12 cells. Hippocampal cultures were prepared as reported previously, ${ }^{11}$ using seven-day old Wistar male rats, and used for experiments at 14 DIV. Differentiated PC12 cells were prepared as described elsewhere. ${ }^{13,24}$

\section{Preparation of DNA carriers and gene gun cartridges}

Gold particles (Bio-Rad, Hercules, CA), $1.6 \mu \mathrm{m}$ in diameter, were coated with plasmid DNA according to the Helios gene gun manufacturer manual (Bio-Rad). The two plasmids used were a cytoplasmic-targeted eGFP and membrane targeted lckGFP, both a gift from Dr Eubanks (Toronto Western Research Institute, University Heath Network, Toronto, ON). lckGFP labels the inner leaflet of plasma membrane and has been previously used to label neurons in slice culture. More details about the lckGFP plasmid and its use are described elsewhere. ${ }^{12,13}$ Briefly, $100 \mu \mathrm{L}$ of $0.05 \mathrm{M}$ spermidine was mixed with $25 \mathrm{mg}$ of gold particles, vortexed, sonicated, and added to $50 \mu \mathrm{g}$ of DNA, to yield a DNA loading ratio (DLR) of $2 \mu \mathrm{g} \cdot \mathrm{mg}^{-1}$ (DLR is the ratio of the amount of DNA in micrograms, to the amount of microcarriers in milligrams). The mixture was vortexed while adding $100 \mu \mathrm{L}$ of $\mathrm{CaCl}_{2}$ drop wise. After incubation for $10 \mathrm{~min}$ at room temperature (to allow plasmid DNA to precipitate on the microcarriers), the microcarrriers were pelleted by centrifugation for $15 \mathrm{~s}$ at $15,000 \mathrm{rpm}$. The carriers were then washed three times with $100 \%$ ethanol and resuspended in $3 \mathrm{~mL}$ of $0.1 \mathrm{mg} \cdot \mathrm{mL}^{-1}$ polyvinylpyrrolidone (PVP) in ethanol. Gene gun cartridges were prepared using the tubing prep station (Bio-Rad). The DNA microcarrier suspension was introduced into $75 \mathrm{~cm}$ of Tefzel ${ }^{\circledR}$ tubing, and allowed to settle for 3-5 min. The PVP solution was then removed and the tubing rotated to spread the microcarriers over the tubing surface. After drying the tubing with nitrogen for 3-5 min, it was cut into 0.5 inch cartridges and these were stored at $4^{\circ} \mathrm{C}$ in a desiccated environment.

\section{Biolistic transfection using Helios gene gun}

Cartridges coated with DNA microcarriers were loaded into the gene gun cartridge holder and a diffusion screen was attached to the tip of the gene gun spacer. To prevent tissue damage and particle clusters, we inserted a filter device between the slice culture and the gene gun nozzle. ${ }^{13}$ 
To construct the filter device, we removed the glass bottom from a $50 \mathrm{~mm}$ (glass bottomed) tissue culture dish and glued a Millipore membrane filter (isopore polycarbonate filter, $3 \mu \mathrm{m}$ pore size, TSTP04700) over the resulting space. Optimal shooting parameters varied with helium gas pressure (80-200 psi) and distance between gun nozzle and the tissue (10-25 mm). For transfection, the culture membranes were removed and 'shot' on a plastic stage, after which they were returned to 6-well dishes for a minimum of 3 days.

\section{Osmotic challenge}

To induce cell swelling and shrinking we used our standard osmotic challenge ${ }^{1-3}$ with some minor modifications. To induce swelling, the culture medium was replaced by a hypoosmotic (swelling) solution (10\%-30\% culturing medium, ie, a 1:9 to 1:7 medium: $\mathrm{H}_{2} \mathrm{O}$ ) for 3.5-4 min. Cell shrinking was induced by rapidly switching from the swelling solution to a normo-osmotic medium (the shrinking solution). Slices were imaged prior to, during, and after solution changes.

\section{OGD challenge}

Membranes with hippocampal slices were washed three times in a glucose-free BSS with $\mathrm{Ca}^{2+}$ and $\mathrm{Mg}^{2+}(116 \mathrm{mM}$ $\mathrm{NaCl} ; 5.4 \mathrm{mM} \mathrm{KCl} ; 0.8 \mathrm{mM} \mathrm{MgSO}_{4}$. $7 \mathrm{H}_{2} \mathrm{O}$; $1 \mathrm{mM} \mathrm{NaH}_{2} \mathrm{PO}_{4}$. $\mathrm{H}_{2} \mathrm{O} ; 1.8 \mathrm{mM} \mathrm{CaCl}_{2} ; 26 \mathrm{mM} \mathrm{NaHCO}_{3}: \mathrm{pH}$ 7.2). To induce glucose deprivation and inhibit glycolysis, the BSS was replaced by BSS with $\mathrm{Ca}^{2+}$ and $\mathrm{Mg}^{2+}$ plus $2 \mathrm{mM}$ 2deoxy-Dglucose (2dDG). To induce hypoxia, cultures were gassed in a chamber with a mixture of $95 \% \mathrm{~N}_{2} / 5 \% \mathrm{CO}_{2}$ at $37^{\circ} \mathrm{C}$. To terminate OGD, the membranes were reperfused with normal culture medium and replaced in an incubator gassed with $5 \% \mathrm{CO}_{2} / 95 \%$ air. Controls (sham OGD) were rinsed with BSS with $575 \mathrm{mM}$ of D-glucose and kept in the incubator gassed with $5 \% \mathrm{CO}_{2}$.

\section{Cell death}

Cell death in slices was assessed by adding $5 \mu \mathrm{L}$ of a $1 \mathrm{mg} \cdot \mathrm{mL}^{-1}$ stock of propidium iodide (PI) to the culture media. PI labels the nuclei of dead cells and is routinely used to evaluate neuronal cell death in slice cultures. ${ }^{11}$ The PI signal was imaged in defined regions across the slice, including CA layers CA1, CA2, CA3, and dentate gyrus (DG), where neuronal cell bodies predominate. Slices were imaged prior to OGD, immediately post-OGD, immediately post-reperfusion, and 24 and $48 \mathrm{~h}$ post-reperfusion. Sham OGD controls were imaged identically. Gains and black levels were standardized for each experiment. At the end of an experiment, the culture membranes were submerged in phosphate buffered solution (PBS) for $24 \mathrm{~h}$ at $4^{\circ} \mathrm{C}$ to produce maximal cell death in the slices. Cell death was expressed as a percentage of maximal cell death as follows: \% cell death $=(\mathrm{Ft} / \mathrm{Fm}) \times 100 \%$, where $\mathrm{Ft}=$ fluorescence at time $\mathrm{t}$, and $\mathrm{Fm}=$ maximal fluorescence.

\section{Confocal microscopy}

Imaging was done on an inverted (Nikon based) scanning confocal microscope (Bio-Rad MRC-600, Bio-Rad, Hercules, CA) equipped with argon-ion laser on an inverted microscope platform. For imaging with the $4 \times, 10 \times$, or $20 \times$ objectives, culture membranes were transferred to a sterile $50 \mathrm{~mm}$ glass bottomed culture dish (WillCo Wells, Amsterdam). For high resolution imaging (using $40 \times$ or $60 \times$ objectives), the plastic feet on the bottom of the culture membranes were removed to reduce the distance between the slices and the bottom of the glass culture dish. ${ }^{13}$ To reduce photo-bleaching, we initially identified transfected neurons at low magnification using epifluorescence with a neutral density filter in place to minimize photo-bleaching. All confocal imaging was done with a neutral density filter of 1 or 2 in place ( $75 \%$ or $50 \%$ transmission respectively) and a zoom factor below 3. Typically neurons were first scanned 2-3 times, two times at a fast scan rate while adjusting the focus, before a final image was collected using a single slow scan (1.5 sec per scan). Cells imaged with both the $20 \times$ and $60 \times$ objectives were scanned a maximum of eight times (six fast; two slow) per time point. Preliminary experiments indicated that this imaging regime caused no detectable photo-bleaching in eGFP or lck neurons (although photo-bleaching could be achieved with one or more of the following: higher zoom factors, more scans, less filtering). After imaging, the culture membranes were transferred back to normal culture dishes. Those membranes now lacking feet were transferred to culture dishes that had glass capillary tubes (diameter $1.5 \mathrm{~mm}$ ) on the bottom; these tubes substituted for the missing feet. For OGD experiments, neurons were typically imaged prior to OGD; immediately post-OGD; immediately post-reperfusion; and $7 \mathrm{~h}, 24 \mathrm{~h}$, and $48 \mathrm{~h}$ post-reperfusion. For experiments that involved OGD plus a subsequent osmotic challenge (swelling and shrinking), neurons were typically imaged pre-OGD; post-OGD; during cell swelling; and immediately to $24 \mathrm{~h}$ after cell shrinking. The majority of experiments used slice cultures, however, a subset of experiments employed dissociated hippocampal neurons and differentiated PC12 cells to determine the effects of an osmotic challenge delivered 1-2 h post-OGD on OGD-induced cell swelling. Controls were subject to 
OGD and a sham osmotic challenge (rinsing in normal media only). In the initial experiments, a blinded observer scored cell bodies in phase microscopy images according to the following morphological criteria: normal; slightly swollen; or severely swollen. In subsequent experiments $(n=3)$, on differentiated PC12 cells only, we evaluated cell body swelling two hours post-osmotic challenge (four hours postOGD) more quantitatively, by measuring cell body width at its widest point. Values were obtained using Biorad software and expressed as a percentage of the control \pm the standard error of the mean (SEM).

\section{Results}

\section{Biolistic transfection of neurons with eGFP and IckGFP in cultured hippocampal slices}

The optimal transfection yield for our experiments was between 10 and 80 neurons per slice. Transfected cells were scattered throughout slice cultures (see Figure 2A), including layers CA1-3 and DG, although the density varied, likely due to uneven distribution of gold particles. Neurons were distinguished from astrocytes on morphological grounds. Cells with a distinct axon and dendrites, or numerous long processes, were classified as neurons and were selected for further experiments. As expected, in neurons transfected with eGFP, which labels the cytoplasm, the signal was distributed throughout the cell and was most intense in the somata (see Figure 2B). eGFP was rapidly lost by dying and dead cells (identified by their PI positive nuclei). lckGFP labels the plasma membrane ${ }^{12,13}$ and in transfected neurons, the fluorescent signal appeared more particulate (see Figure 2C). In contrast to eGFP, lckGFP was retained in the plasma membrane for hours to days after cell death. Each plasmid offered specific experimental advantages. Neurons transfected by lckGFP (lckGFP neurons) allowed us to image swelling and shrinking in the plasma membrane directly, whereas neurons transfected with eGFP (eGFP neurons) allowed us to monitor changes in cytoplasmic volume, and to confirm cell viability. Consequently, both plasmids were used in subsequent experiments. Biolistic transfection showed low toxicity $^{12-14}$ and both eGFP and lckGFP were retained in live neurons for up to 4 weeks.

\section{Swelling and shrinking neurons in hippocampal slice cultures}

To study neuronal capacity to swell and shrink, hippocampal slices were subjected to our standard osmotic challenge (changes from normo-osmotic, to hypo-osmotic, to normo-osmotic media). In preliminary experiments, the majority of eGFP neurons (11/12 cells in two slices), and lckGFP neurons (10/12 cells in two slices), swelled rapidly in response to hypo-osmotic medium, and then rapidly shrank after a return to normo-osmotic media. In some lckGFP neurons, the fluorescent signal in the plasma membrane appeared to fade during cell swelling, and then recover, as neurons shrank. Subsequent experiments followed 14 eGFP neurons (in seven slices, in three separate experiments), and nine lckGFP neurons (in three slices, three experiments) in detail. Within 1-4 min of switching to hypotonic media, cell bodies swelled and extensive neurite beading was evident (Figure 2: see D2, E2, F2). Upon switching to normo-osmotic medium, neurites rapidly lost their beaded appearance, cell bodies shrank, and within five min, most neurons had regained their pre-swell morphology (Figure 2: see D3, E3). Real-time imaging demonstrated that neurons could swell and shrink repeatedly in response to multiple cycles of swelling and shrinking solutions (Figure 2: see F1-F5). VLDs were not observed during neuronal shrinking. Previous studies on dissociated cells ${ }^{1-5}$ have shown that VLDs are initiated at adherent surfaces as pinpoint invaginations that rapidly dilate and balloon upward into the cytoplasm, before rapidly closing, or collapsing (within seconds to min). These characteristics make them difficult to detect in neurons in slice cultures. This is particularly true when only the cytoplasm is labeled; under such conditions, VLDs appear as transient, black holes that lack a discernable membrane (Figure 1J and Supplementary Figure B-D). ${ }^{1}$ To confirm that differentiated hippocampal neurons could in fact form VLDs, we used high density multi-layered dissociated hippocampal cultures at 14 days in vitro and monitored neuronal swelling and shrinking in response to our standard osmotic challenge $(n=4$ cultures, from four separate rats). As shown in Figure $1 \mathrm{G}$, VLDs rapidly formed in shrinking hippocampal neurons and subsequently disappeared during the VLD recovery phase.

\section{Establishing sublethal OGD in hippocampal slices}

To establish a mild and a severe ischemic insult, we evaluated cell death in slices after 15, 30, and $60 \mathrm{~min}$ of OGD. OGD for $60 \mathrm{~min}$ did not significantly increase cell death immediately post-OGD versus sham OGD controls. However, by $48 \mathrm{~h}$ post-reperfusion, cell death increased to $67 \%-73 \%$ of maximal values compared to sham OGD controls, which were $16 \%-22 \%$ of maximal values ( $P<0.001 ; \mathrm{n}=16 \mathrm{regions} /$ slice and $\mathrm{N}=3$ slices $).$ Cell death occurred in all regions of the slice, but some trans- 

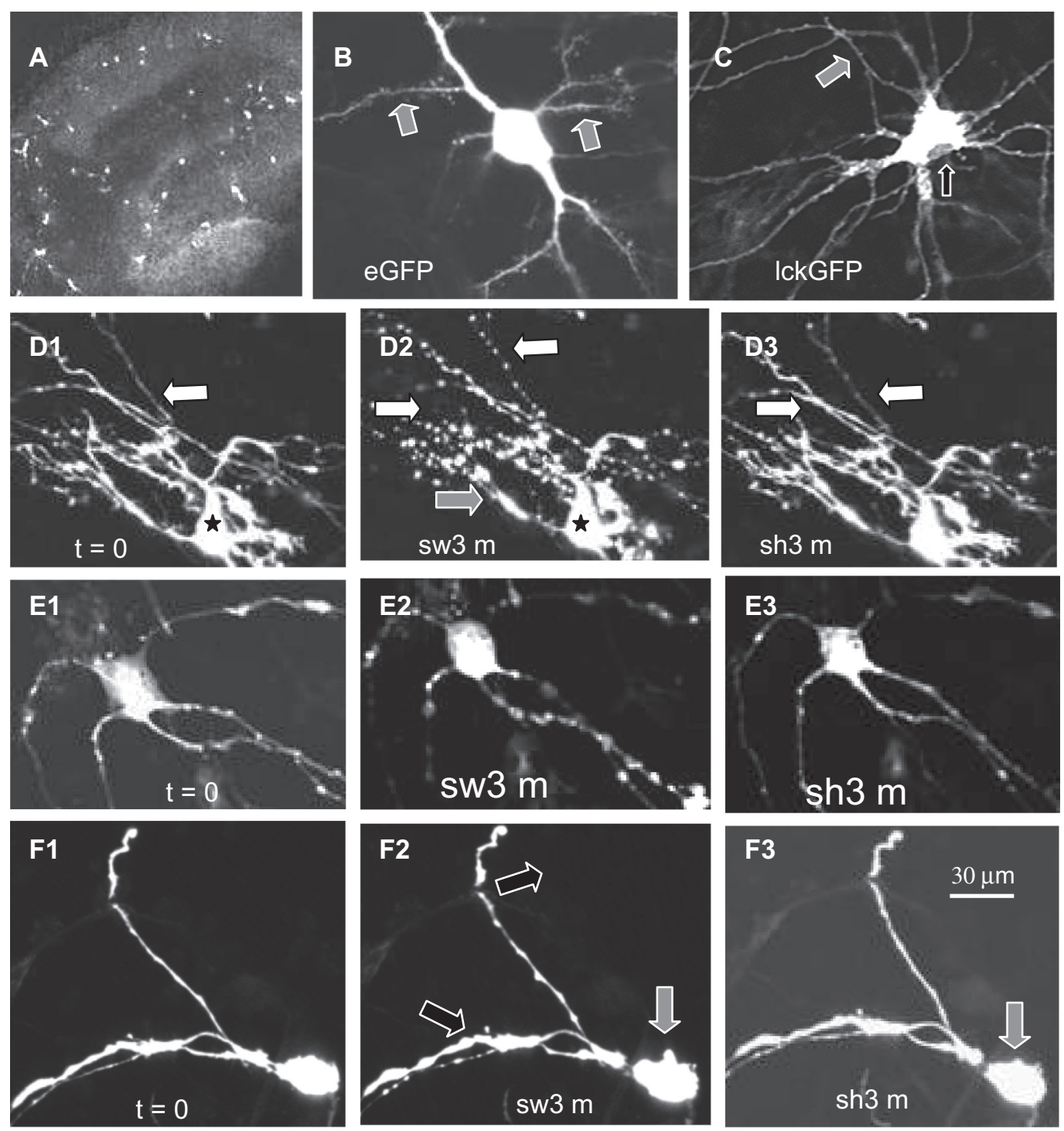

Cycle 2
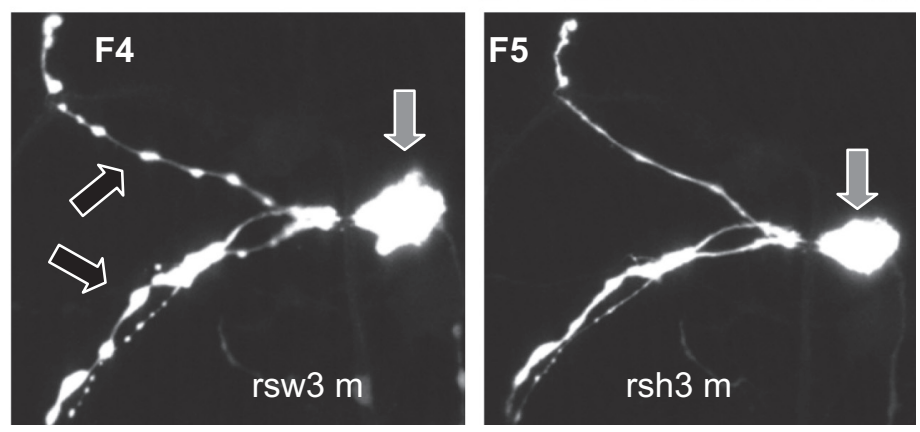

Figure 2 Imaging neurons transfected with eGFP and IckGFP plasmids in hippocampal slice cultures. A is a low magnification (4x) image of a slice transfected with eGFP. Bright spots are transfected cells. B is an eGFP transfected pyramidal neuron; the cell body and multiple dendrites are labeled and spines are visible along the dendrites (gray arrows). C is a IckGFP transfected neuron. In this image, the unlabelled nucleus is visible (black arrow) and spines are evident along dendrites (gray arrow). B and $\mathbf{C}$ are merges of two confocal images through different focal planes. Slices were isolated from 7-day-old rats, cultured for $\mathbf{7}$ days prior to transfection, and imaged 2 days post-transfection (see methods). DI-D3 show two eGFP transfected neurons in a hippocampal slice culture. DI shows pre-swell; the soma of the larger neuron is labeled with a black star and extensive dendrites are visible (eg, see white arrow). Within 3 min of the transfer to hypo-osmotic media, a slight swelling (sw) of the cell body (see black star in D2) occurs, but dendritic swelling is marked (gray arrow), and dendrites undergo a massive transformation to a 'beads on a string' morphology (white arrows). Three min after a return to normo-osmotic medium, the beads on a string morphology are rapidly being lost as dendritic morphology is restored to near normal (see white arrows on D3) and the soma begins to shrink (sh). EI-E3 show an IckGFP transfected neuron in a hippocampal slice, time course matched to DI-D3. EI is pre-swell and E2 shows how after exposure for $3 \mathrm{~min}$ to the swelling solution, dendritic beading occurs. After the return to normal media the cell shrinks and dendritic beading is lost (E3). In FI-F5, an eGFP labeled neuron is imaged over two cycles of swelling and shrinking. The cell body (gray arrow) swells in hypo-osmotic media and shrinks in a normo-osmotic media response to swelling solution. Dendritic beading (black arrows) is more pronounced in cycle two. 
fected neurons did survive for up to 2 days. OGD for 30 min caused a similar delayed pattern of cell death and values $48 \mathrm{~h}$ post-OGD were not significantly different from OGD for $60 \mathrm{~min}$. OGD for $15 \mathrm{~min}$ caused no immediate cell death and only slightly increased cell death at $48 \mathrm{~h}$ versus controls $(12 \%-28 \%$ of maximal versus $8 \%-13 \%$ of maximal; $P<0.001 ; \mathrm{n}=16$ regions/slice and $\mathrm{N}=4$ slices. Based on these results, $15 \mathrm{~min}$ of OGD was classified as mild OGD, and both $30 \mathrm{~min}$ or $60 \mathrm{~min}$ of OGD as severe OGD.

\section{OGD-induced neuronal swelling and recovery}

Mild OGD (15 min) caused minimal-to-no detectable swelling of eGFP neurons imaged immediately after removal from the anoxic chamber. However, within five min of reperfusion (the re-introduction of normal media), swelling of cell bodies; thickening of some processes; and minor dendritic beading was apparent in 5/8 neurons examined (eg, Figure 3C). Mild OGD caused similar changes in lckGFP neurons, however, in most cases (12/15 cells in three slices), the degree of neurite beading appeared more pronounced than in eGFP neurons.

Following reperfusion, cultures were returned to the 5\% $\mathrm{CO}_{2}$ incubator for up to $48 \mathrm{~h}$. At 7 hours post-reperfusion, most $(6 / 8)$ eGFP neurons $(n=2$ slices $)$ still showed some degree of somal swelling and mild neurite beading.
In 5/8 neurons, multiple VLD-like structures were observed (see Figure 3D' and Figure 4). These VLDs appeared as dark holes in the cytoplasm (Figure 3D and Figure 4A, B, $\mathrm{G}, \mathrm{I}, \mathrm{J})$, consistent with VLD appearance in dissociated cells under conditions where the cytoplasm is labeled (see Figure $1 \mathrm{~J}$ and Supplementary Figure B-D). In 4/8 neurons, VLDs persisted for up to $24-48 \mathrm{~h}$ post-reperfusion, indicating that VLD recovery had stalled. In lckGFP neurons, VLDs were also observed post-OGD and VLD recovery also appeared to stall; in 6/10 neurons, VLDs were observed as long as 48 $\mathrm{h}$ post-OGD. In lckGFP neurons, VLDs appeared as fluorescent rings (Figure 4C-E), consistent with VLD morphology in dissociated cells where the plasma membrane is labeled (see Figure 1C, C' and Supplementary Figure G, H). In contrast to VLDs, neurite beading in eGFP or lckGFP neurons rarely persisted beyond $24 \mathrm{~h}$ post-OGD. Some neurons recovered fully from swelling by $24 \mathrm{~h}$ post-OGD, but notably, even neurons that remained swollen were capable of generating new neurite outgrowth (see for example Figure 3E). Sham OGD controls ( $\mathrm{n}=12$ cells, in four slices, in three separate experiments for $\mathrm{eGFP}$ neurons, and $\mathrm{n}=9$ cells in three slices, in three separate experiments for lckGFP neurons) showed no swelling over the same time period.

More severe OGD (30 and $60 \mathrm{~min}$ ), evoked only mild swelling in most eGFP transfected neurons (22/23),
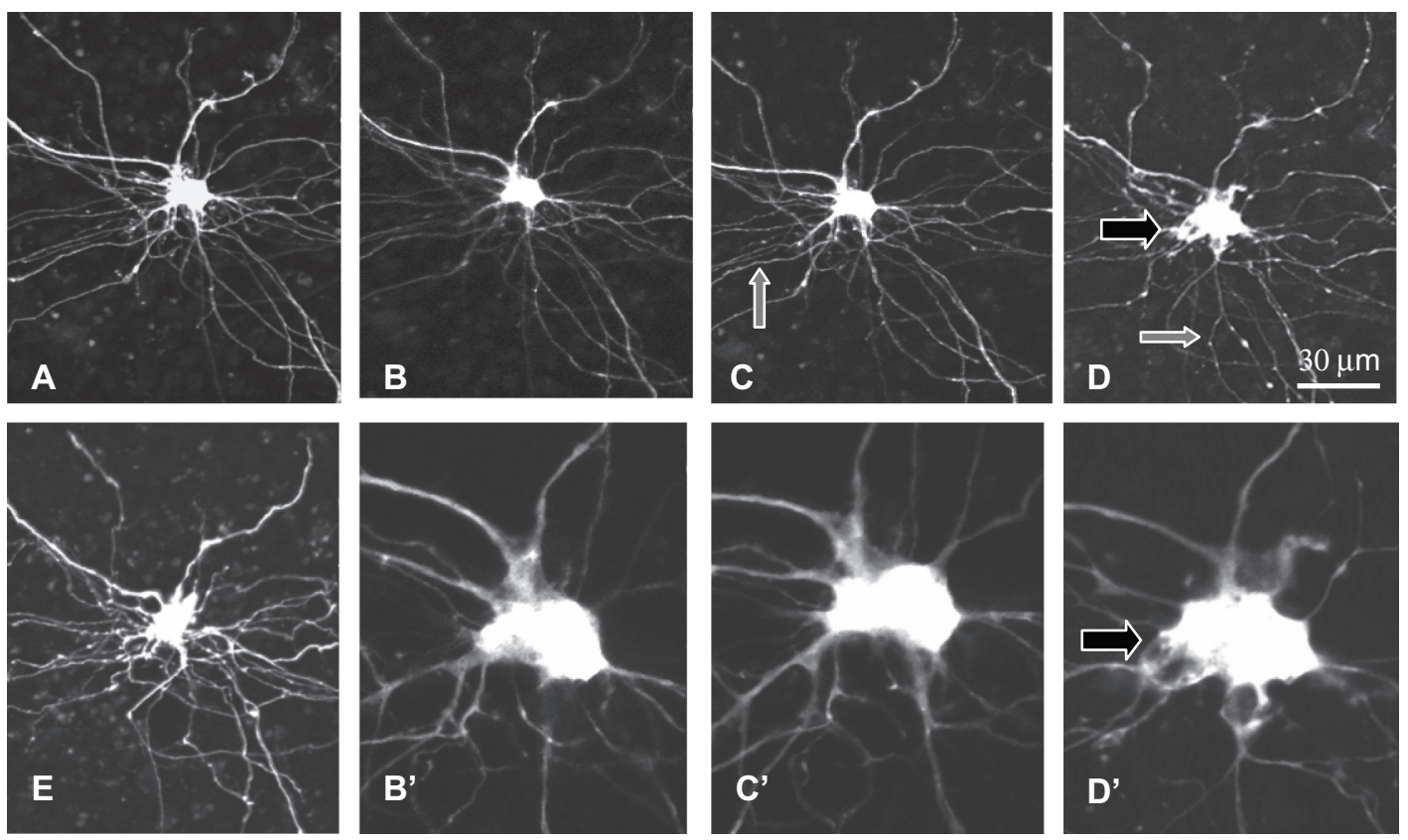

Figure 3 OGD-induced changes in an eGFP transfected hippocampal neuron imaged over two days. B'-D' is higher magnification of the same cell shown in A-E. A is prior to OGD for $15 \mathrm{~min}$. B and B' are immediately post-OGD. C and C' are $10 \mathrm{~min}$ post-reperfusion; the soma is swollen and mild dendritic beading is evident (gray arrows). $\mathbf{D}$ and $\mathbf{D}$ ' show that by 7 hours post-OGD, the swelling of the cell body is more pronounced, as is dendritic beading. VLD like structures are visible, see black arrow in $\mathbf{D}$ '. In $\mathbf{E}, 48 \mathrm{~h}$ after reperfusion, the soma remains swollen but the neuron is alive (PI negative, with no cytoplasmic leaking of eGFP); comparison with $\mathbf{A}$ shows new outgrowth has occurred. 

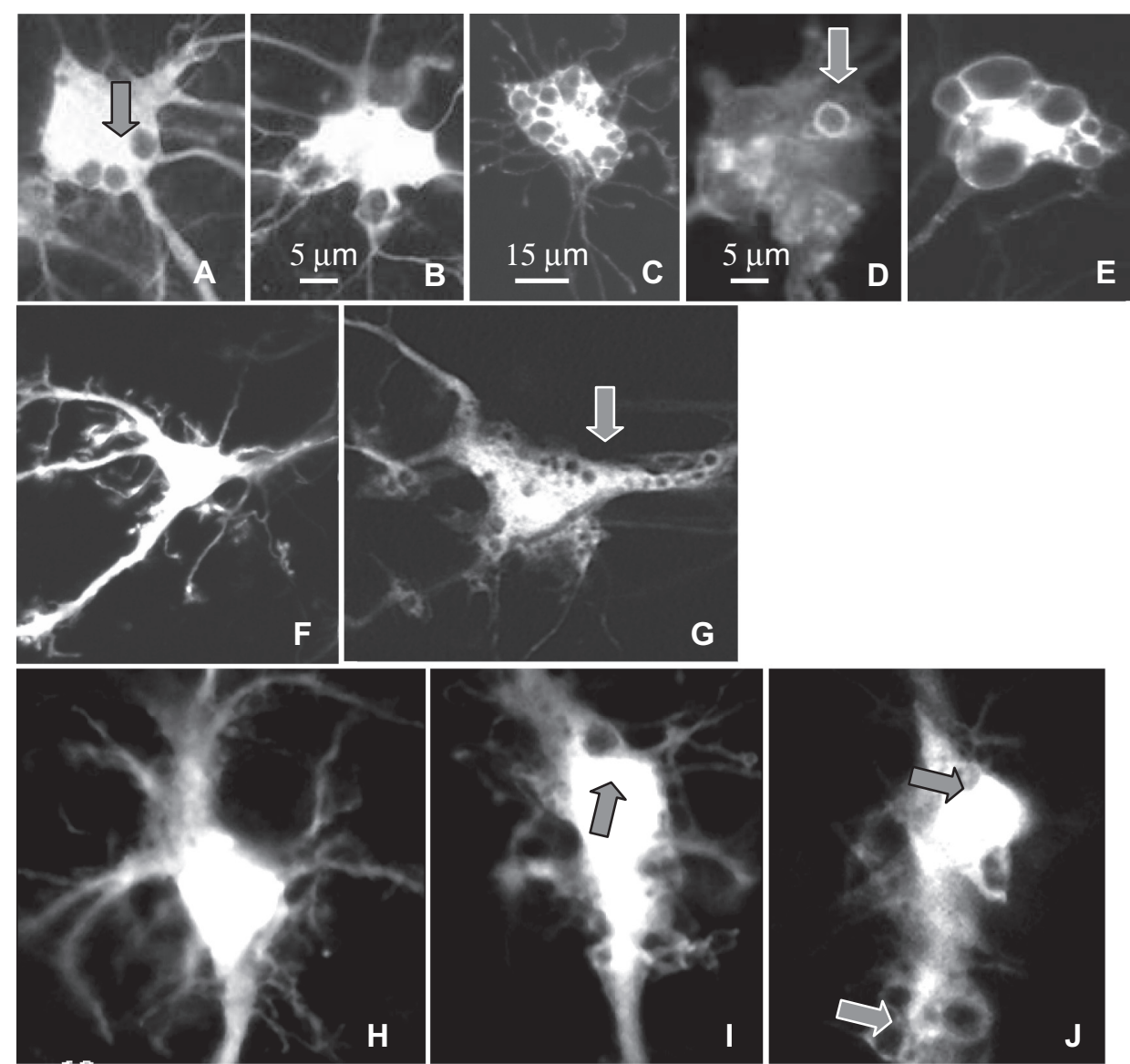

Figure 4 A-E Neurons develop VLD-like structures post-OGD. A-C are the eGFP neurons; $\mathbf{A}$ and $\mathbf{B}$ are $7 \mathrm{~h}$ post-OGD and $\mathbf{C}$ is $48 \mathrm{~h}$ post-OGD. D and $\mathbf{E}$ are the Ick-GFP neurons, 24 and $48 \mathrm{~h}$ post-OGD respectively. The arrow labels VLD, which appears as a ring. Compare to VLDs in IckGFP labeled PCI 2 cells (Figure I and Supplementary Figure). F and $\mathbf{G}$ are both merges of two confocal planes of an eGFP neuron; $\mathbf{F}$ is pre-OGD and $\mathbf{G}$ is $7 \mathrm{~h}$ post-OGD. $\mathbf{G}$ is shown slightly enlarged relative to $\mathbf{F}$, to show VLDs. H-J show an eGFP transfected neuron, prior to OGD for $30 \mathrm{~min} ; 7 \mathrm{~h}$ post-OGD; and $48 \mathrm{~h}$ post-OGD. Gray arrows in I and J label VLDs.

immediately post-OGD. However, many of these neurons continued to swell post-reperfusion, and $9 / 23$ died within the next $48 \mathrm{~h}$ (see Figure 5A-D). In 4/14 neurons that survived, VLDs were observed by 7 hours post-OGD, and these VLDs persisted for $24-48 \mathrm{~h}$ indicating that VLD recovery was stalled. In lckGFP neurons (14/18 in three slices), cell swelling and dendritic beading was apparent immediately post-OGD (Figure 5E-G) and, as was the case with mild OGD, appeared to be more pronounced than in the eGFP neurons. In those neurons that subsequently died, dendritic beading was sustained, unlike the rapid recovery from dendritic beading observed in response to osmotic downshock (compare Figure 5E-G with $\mathrm{H}-\mathrm{J}$ ), or the recovery after mild OGD. As shown in Figure 5 (see K-M), sham OGD had no effect on control eGFP neurons ( $9 / 9$ cells in three slices) or on lckGFP neurons ( $9 / 10$ cells, data not shown).

To determine if OGD impaired neuronal capacity to recover from a subsequent swelling episode, we subjected slices to OGD, and 1-2 h post-reperfusion delivered our standard osmotic challenge. A prior episode of mild OGD (15 min) had no detectable effect on neuronal responses to an osmotic challenge. Both eGFP (8/8 neurons) and lck neurons (4/4) swelled rapidly in response to hypo-osmotic medium, and shrank almost immediately after a return to normo-osmotic medium. As shown in Figure 6, even severe OGD (30 min) did not impair neuronal capacity to swell and shrink repeatedly in response to sequential osmotic challenges delivered 1-2 $\mathrm{h}$ post-OGD. In 4/8 neurons subject to severe OGD, recovery of normal (pre-OGD) morphology appeared subjectively to be more robust compared to neurons subjected to OGD only. A similar effect was observed in differentiated PC12 cells and dissociated hippocampal neurons subjected to $30 \mathrm{~min}$ of OGD. In cultures given an osmotic challenge 2 hours after OGD (30 min), the extent of cell swelling evaluated subjectively 2 hours later was judged to be less severe than in control cultures subjected 

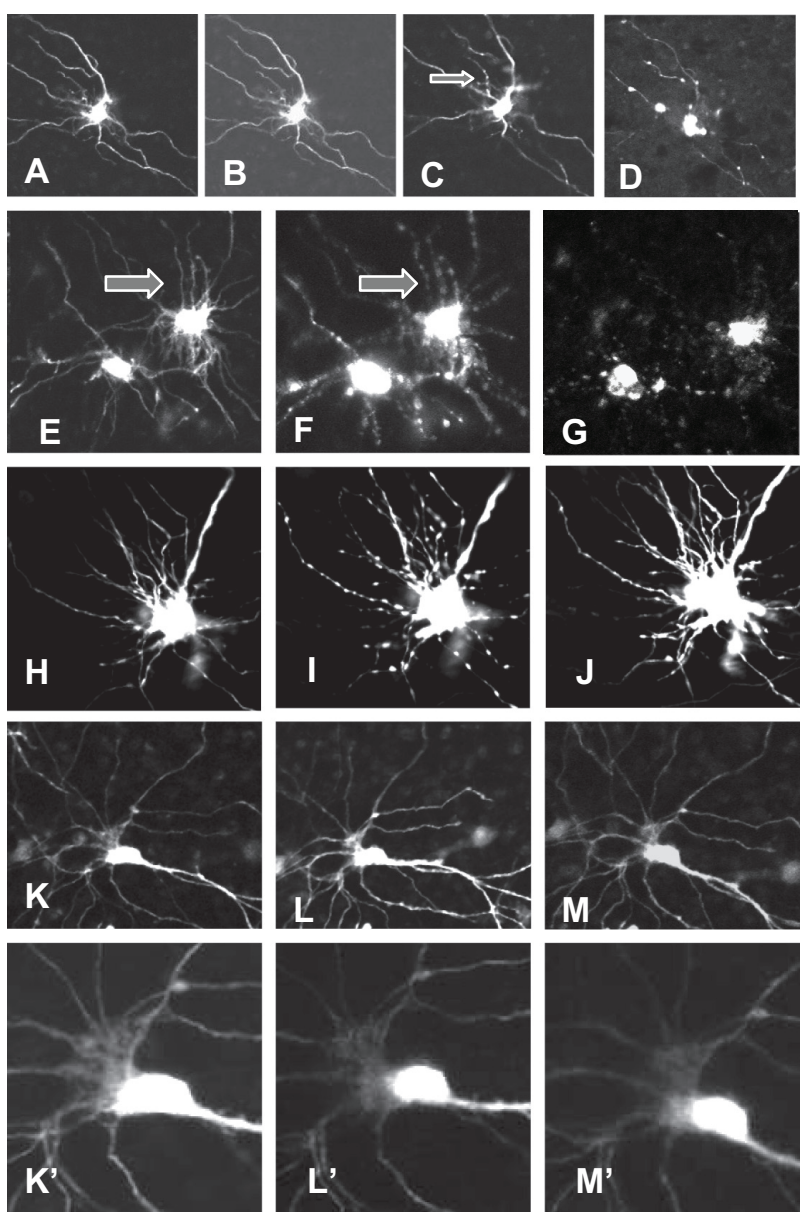

Figure 5 Morphological changes in eGFP (A-D) and IckGFP (E-G) labeled hippocampal neurons, after severe OGD (30 min). A is prior to OGD, B is immediately post-OGD, $\mathbf{C}$ is $7 \mathrm{~h}$ post-OGD (gray arrow points to where some beading is evident) and $\mathbf{D}$ is $48 \mathrm{~h}$ after reperfusion when the neuron has just died. $\mathbf{E}$ is prior to OGD; no dendritic beading is evident (gray arrow). $\mathbf{F}$ is immediately post-OGD; extensive neurite beading (gray arrow) and somal swelling is evident. G is $48 \mathrm{~h}$ post-OGD; the neurons have not recovered and have died. $\ln \mathbf{H}-\mathbf{J}, \mathbf{H}$ is pre swell, $\mathbf{I}$ is after $2 \mathrm{~min}$ in swelling solution, and $\mathbf{J}$ is within I min of the transfer to normal media; dendrites show significant recovery from the extensive beading. K-M show a control eGFP transfected neuron treated with sham-OGD. K'-M' is the same neuron at higher magnification. $\mathbf{K}$ and $\mathbf{K}^{\prime}$ show the pre-rinse with normal media (sham-OGD), $\mathbf{L}$ and L' are immediately post-reperfusion, and $\mathbf{M}$ and $\mathbf{M}$ ' are $7 \mathrm{~h}$ post-sham OGD.

to OGD or glucose deprivation (GD) plus a sham osmotic challenge. To address this issue more quantitatively, we used phase optics to measure cell body width at its widest point in differentiated PC12 cells subject to OGD plus or minus our standard osmotic challenge. Cells previously subject to OGD swelled rapidly in response to hypo-osmotic medium, and then subsequently shrank in response to a return to normo-osmotic media. In many cells, numerous VLDs formed as cells shrank. Two hours later, cell body width in cells that had received the osmotic challenge was reduced by $22 \pm 7 \%$ compared to control cells subject to OGD/sham osmotic challenge $(n=30$ cells in three separate cultures for OGD/osmotic challenge; $\mathrm{n}=30$ cells in three separate cultures for OGD/sham challenge, $P<0.001)$. At this time, numerous VLDs were also observed indicating that recovery of the VLDs that had formed two hours previously as cells shrank in response to the osmotic downshock, had markedly slowed or stalled.

\section{Discussion}

Understanding the cellular responses involved in recovery from neuronal swelling induced by pathophysiological situations, such as cerebral ischemia and brain trauma, is critical. ${ }^{15,22}$ Our previous studies on dissociated neurons have shown that swelling and shrinking neurons reorganize their plasma membrane to accommodate changes in cell surface area. ${ }^{1-5}$ Investigations on such events in vivo have been limited, since CNS neurons must remodel their plasma membrane in the context of a highly restricted extracellular space and synaptic contacts with other neurons. How such accommodation is achieved is also hampered by the difficulty of experimentally evoking plasma membrane remodeling in vivo. To address these issues, we used neonatal hippocampal slice cultures which maintain neuronal architecture ${ }^{11,16}$ and synaptic activity in vitro. ${ }^{16}$

Our results demonstrate that neurons in hippocampal slice culture respond to hypo-osmotic media by rapidly swelling, and upon a return to normo-osmotic media, rapidly shrink. These results are consistent with data obtained from snail and mammalian neurons in dissociated cultures, ${ }^{1-3}$ but in contrast to previous lower resolution studies on slice cultures which showed neuronal swelling over a similar time course (approximately $3.5 \mathrm{~min}$ ), but no recovery (shrinking) postreperfusion. ${ }^{9}$ The fact that we observed almost immediate shrinking after return to normo-osmotic medium suggests that our method of monitoring cell swelling and shrinking may be more sensitive, particularly when the plasma membrane is labeled.

Our experimental paradigm clearly evoked neuronal swelling and shrinking in slice cultures, but the degree to which neurons in vivo swell during osmotic stress is contentious. Previous studies reported that neuronal volume in acute cerebral cortex slices was resistant to osmolarity changes, ${ }^{8}$ although these same neurons swelled in response to OGD. The discrepancy with our results may reflect differences in the magnitude or duration of the osmotic shifts, since those studies used smaller shifts for longer durations. The global osmotic shifts we used are non physiological, however the fact that they reliably evoke swelling and shrinking argues that the machinery that regulates cell surface area is intrinsic 

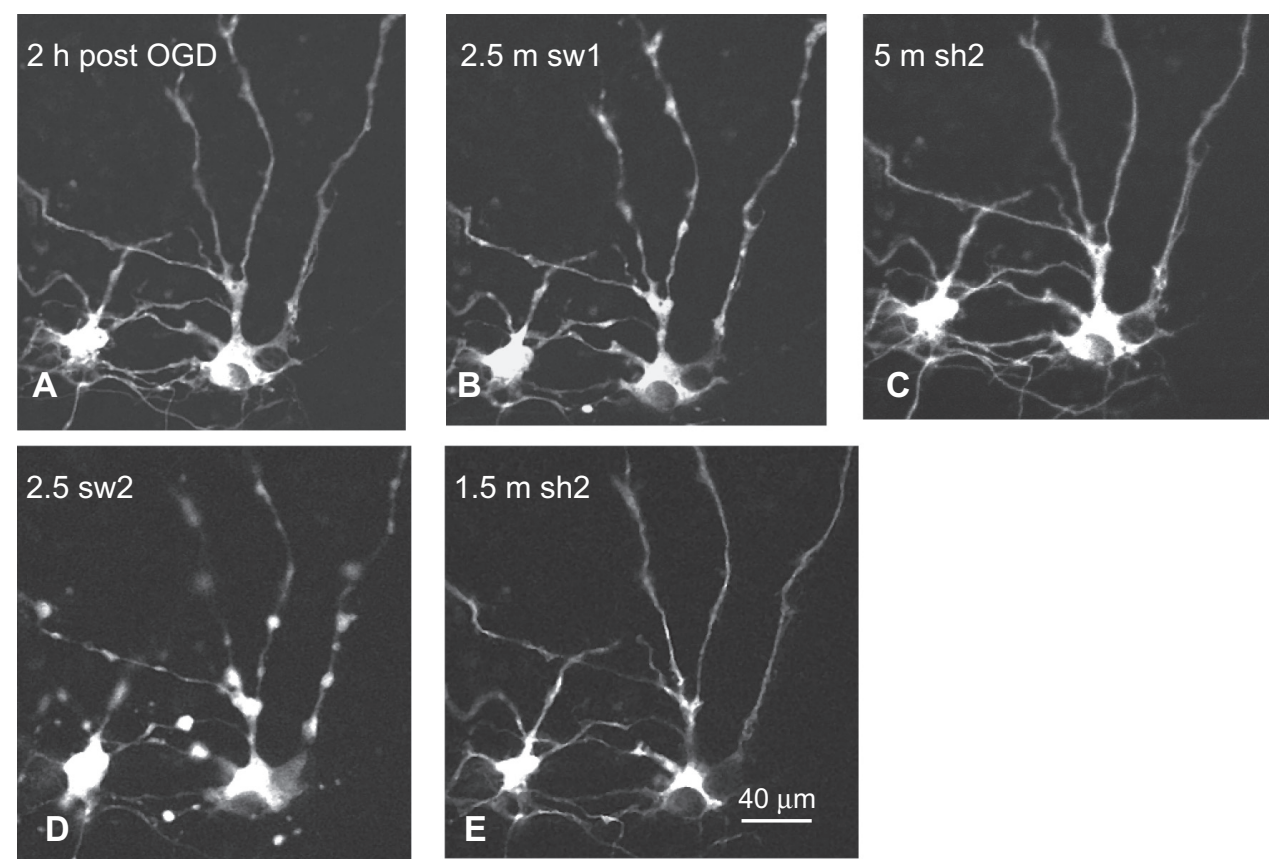

Figure 6 OGD does not impair neuronal capacity to swell and shrink. A-E shows two eGFP neurons challenged with two cycles of swelling and shrinking, $2 \mathrm{~h}$ after severe (30 min) OGD/reperfusion. Times shown are min in swelling (sw) or shrinking (sh) solutions. A is $2 \mathrm{~h}$ post-OGD; both neurons show no dendritic beading but are slightly swollen. In $\mathbf{B}$, dendritic beading occurs during swelling but as neurons shrink, in $\mathbf{C}$, dendrites rapidly recover. $\mathbf{D}$ and $\mathbf{E}$ show how a second cycle induces beading and recovery. In E, the somata have shrunk to pre-OGD size.

to neurons, although it presumably has other physiological roles.

Precisely how water enters neurons in response to a hyposmotic challenge, or OGD, is not clear. Previous studies have provided evidence that many neurons lack functional aquaporins $^{8,17}$ (but see ${ }^{18,19}$ ) and suggested that water must enter by other channels. In our studies, the failure of a few neurons to swell in response to hypo-osmotic stimuli is consistent with previous studies that noted some isolated CA1 pyramidal cells failed to swell in response to a hypotonic challenge. ${ }^{20}$ Such resistance could be due to differences in water permeability, or mechanical resilience, a function of the cytoskeleton which is known to play a role in SAR in dissociated neurons. ${ }^{1-5}$ Further, in the 3D hippocampal slice, mechanical support from adjacent cells and the interstitial matrix, coupled with limited intracellular space, could also prevent some neurons from swelling, at least in the dimensions we monitored.

The shrinking we observed after a return to normoosmotic media indicates that neurons in slice cultures can rapidly recover from swelling. In particular, the recovery of dendrites indicates that dendrites have a remarkable capacity to remodel their plasma membrane. We did not observe VLD formation during shrinking, likely because VLDs are initiated at adherent surfaces as pinpoint invaginations that dilate and balloon upward over seconds, and then rapidly close, or collapse (seconds to min). These characteristics make them difficult to detect in neurons in a $3 \mathrm{D}$ environment like the slice cultures, particularly when the label is cytoplasmic. The fact that dissociated hippocampal neurons in high density, multi-layered cultures developed VLDs during shrinking, and that these VLDs subsequently disappeared, indicates that hippocampal neurons do have the cellular machinery to drive VLD formation/retrieval.

Our results indicate that neurons swell in response to transient OGD/reperfusion. However, most neurons showed only minor swelling immediately after mild OGD, although swelling continued after reperfusion. Previous EM studies also reported minimal change in morphology in hippocampal slice cultures after a similar duration of OGD. ${ }^{7}$ In many neurons transfected with lckGFP, dendritic beading was evident immediately post-OGD, which suggests that the plasma membrane is a more sensitive indicator of cell swelling than cytoplasmic volume. Full recovery from swelling (shrinking) occurred slowly and was frequently incomplete for up to two days. This time course likely reflects the fact that even mild OGD/reperfusion evokes a complex response that includes ATP depletion, increased free radicals, and prolonged depolarization. Dendrites recovered from swelling relatively quickly and dendritic beading was rarely observed after 24 $\mathrm{h}$ post-OGD. Even after severe OGD, dendritic beading was not observed after $24 \mathrm{~h}$ except in dying cells. This recovery 
is further evidence that dendrites have considerable capacity to recover from swelling and is consistent with studies in vivo showing that, even after severe ischemia, dendritic structure can partially recover if reperfusion occurs early enough. ${ }^{21}$

At seven hours post-OGD, VLD-like structures were observed in both eGFP- and lckGFP-transfected neurons. While it was not possible in slice culture to confirm that these structures formed at adherent surfaces, a defining feature of VLDs, their appearance was consistent with VLDs observed in isolated neurons in dissociated cultures, and in neurons in high density multi-layered dissociated cultures. The fact that the VLDs appeared as holes in the cytoplasm in the eGFP transfected neurons, and as rings in the lckGFP transfected neurons, as they do in disassociated neurons labeled with cytoplasmic or plasma membrane markers respectively, also supports this conclusion. In some neurons, VLD recovery appeared to be stalled, since VLDs persisted for up to $48 \mathrm{~h}$ post-OGD. Previous studies have shown that VLD recovery, but not VLD formation, is actin and myosin dependent; in neurons treated with cytochalasin or $N$-Ethylmaleimide (NEM), VLDs can persist indefinitely ${ }^{2}$ and in differentiated PC12 cells, VLD recovery is sensitive to ATP depletion, and disruption of actin polymerization. ${ }^{22}$ Whether ATP levels or actin are affected in those neurons in slice where VLD recovery was stalled, remains to be determined.

Our results differ from previous studies reporting rapid swelling and extensive dendritic beading immediately after 15 min of OGD..$^{21}$ These studies also reported no recovery after OGD, but since these neurons were imaged only immediately post-OGD, later stages of recovery could have been missed. Since we also observed immediate swelling and extensive neurite beading immediately after severe OGD, it seems likely that the question is one of degree; neurons in our cultured slices may be relatively more resistant to OGD and more capable of recovery after reperfusion.

In hippocampal slices, neuronal capacity to recover from swelling was not impaired by a prior episode of OGD. Neurons swelled and shrank in response to up to three sequential cycles of osmotic stress, suggesting that neuronal capacity for SAR was unaffected by OGD at least in the first 5 hours post-reperfusion. Even after severe OGD (30 or $60 \mathrm{~min}$ ), neurons could recover from cell swelling induced by hypoosmotic media. Indeed, recovery of normal (pre-OGD) morphology appeared to be more robust in 4/8 neurons compared to control neurons subjected to OGD only. Similar effects were observed in primary neurons and differentiated PC12 cells; those given an osmotic challenge 1-3 h after OGD (30 $\mathrm{min})$ appeared subjectively to be less swollen than controls subject to OGD/sham osmotic challenge (rinsing in normal media). Cell body swelling in differentiated PC12 cells evaluated by more quantitative methods showed that cell body diameter was significantly reduced in cells subject to an osmotic challenge within two hours of OGD, compared to OGD alone controls. These results suggest that the rapid forced shrinking induced by the osmotic downshock can promote recovery from OGD-induced swelling. As such, they have important implications for therapies directed at reducing neuronal swelling induced by ischemia and brain trauma.

Our results showing that mammalian neurons in a 3D environment can recover from swelling induced by osmotic stress and OGD strongly suggest that SAR is an intrinsic property of neurons. What then is the physiological role of SAR? Whenever neurons change their shape, or volume, remodeling of the plasma membrane is necessary. Thus, during neuronal development, SAR will be obligate during cell migration, growth-cone pathfinding, and neurite outgrowth. SAR is also required for growth cone remodeling during regeneration. ${ }^{23}$ Neuronal capacity for SAR may be a critical determinant of how successfully the brain can respond to insults like excitotoxicity; mechanical trauma; epilepsy; and ischemia, that involve cell swelling; disruptions of the cytoskeleton; and loss of cell-cell contacts. Further, since propagation of the electrical signal decreases with increased surface area ${ }^{4}$ SAR may help preserve functional recovery, as well as neuronal survival.

\section{Disclosure}

The authors disclose no conflicts of interest.

\section{References}

1. Mills LR, Morris CE. Neuronal plasma membrane dynamics evoked by osmomechanical perturbations. J Membr Biol. 1998;166: 223-235.

2. Reuzeau C, Mills LR, Harris JA, Morris CE. Discrete and reversible vacuole-like dilations induced by osmomechanical perturbation of neurons. J Membr Biol. 1995;145:33-47.

3. Herring TL, Cohan CS, Welnhofer EA, Mills LR, Morris CE. F-actin at newly invaginated membrane in neurons: implications for surface area regulation. J Membr Biol. 1999;171:151-169.

4. Morris CE, Homann U. Cell surface area regulation and membrane tension. J Membr Biol. 2001;179:79-102.

5. Morris CE, Wang JA, Markin VS. The invagination of excess surface area by shrinking neurons. Biophys J. 2003;85(1):223-235.

6. Andrew RD, Macvicar BA. Imaging cell volume changes and neuronal excitation in the hippocampal slice. Neuroscience. 1994;62(2): 371-383.

7. Lushnikova IV, Voronin KY, Malyarevskyy PY, Skibo GG. Morphological and functional changes in rat hippocampal slice cultures after short-term oxygen-glucose deprivation. J Cell Mol Med. 2004; $8(2): 241-248$ 
8. Andrew RD, Labron MW, Boehnke SE, Camduff L, Kirov SA. Physiological evidence that pyramidal neurons lack functional water channels. Cerebral Cortex. 2007;17(4)787-802.

9. Nakajima R, Nakamura T, Ogawa M, Miyakawa H, Kudo Y. Novel method for quantification of brain swelling in rat hippocampal slices. J Neurosc Res. 2004;76:723-733.

10. Adamchik Y, Frantseva MV, Weisspapir M, Carlen PL, Perez Velazquez JL. Methods to induce primary and secondary traumatic damage in organotypic hippocampal slice cultures. Brain Res Protocols. 2000;5:153-158.

11. Feeney C, Frantseva MV, Carlen PL, et al. Vulnerability of glial cells to hydrogen peroxide in cultured hippocampal slices. Brain Research. 2008;1198:1-15.

12. Benediktsson AM, Schachtele SJ, Green SH. Dailey ME. Ballistic labeling and dynamic imaging of astrocytes in organotypic hippocampal slice cultures. J Neurosci Methods. 2005;141(1):41-53.

13. Shulyakova N, Kaifosh P, Mills LR. Cell surface membrane remodeling and mitochondrial remodeling in live neurons: practical approaches for studying dynamic processes using confocal microscopy. In: MendezVilas A, Diaz Alvarez J, editors. Modern Research and Educational Topics in Microscopy. Badajoz, Spain: Formatex Microscopy Book Series; 2007:49-59.

14. Wirth MJ, Wahle P. Biolistic transfection of organotypic cultures of rat visual cortex using a hand-held device. J Neurosci Methods. 2003;125:45-54.

15. Won SJ, Kim DY, Gwag BJ. Cellular and molecular pathways of ischemic neuronal death. J Biochem Mol Biol. 2002;35(1):67-86.

16. Finley M, Fairman D, Liu D, Wood A, Cho S. Functional validation of adult organotypic cultures as an in vitro model of brain injury. Brain Research. 2004;1001:123-132.
17. Agre P, King LS, Yasui M, et al. Aquaporin water channels - from atomic structure to clinical medicine. J Physiol. 2002;542:3-16.

18. Venero JL, Vizuete ML, Ilundain AA, et al. Detailed localization of aquaporin-4 messenger RNA in the CNS: preferential expression in periventricular organs. Neuroscience. 1999;94:239-250.

19. Badaut J, Petit JM, Brunet JF, et al. Distribution of aquaporin 9 in the adult rat brain: preferential expression in catecholaminergic neurons and in glial cells. Neuroscience. 2004;128:27-38.

20. Aitken PG, Borgdorff AJ, Juta AJ, et al. Volume changes induced by osmotic stress in freshly isolated rat hippocampal neurons. Pflugers Arch-Eur J Physiol. 1998;436:991-998.

21. Zhang S, Boyd J, Delaney K, Murphy TH. Rapid reversible changes in dendritic spine structure in vivo gated by the degree of ischemia. J Neuroscience. 2008;25(22):5333-5338.

22. Morris CE, Shulyakova N, Mills LR. Neuronal membrane remodeling after a rapid osmo trauma. Program No. 148.19/I36. Neuroscience 2009: Online Neuroscience Meeting Planner for the 39th Annual Meeting of the Society for Neuroscience; 2009 Oct 17-21; Chicago, IL.

23. Bonanomi D, Fornasiero EF, Valdez G, et al. Identification of a developmentally regulated pathway of membrane retrieval in neuronal growth cones. J Cell Sci. 2008;121:3757-3769.

24. Sirk D, Zhu Z, Wadia JS, et al. Chronic exposure to sub-lethal beta-amyloid (Abeta) inhibits the import of nuclear-encoded proteins to mitochondria in differentiated PC12 cells. J Neurochem. 2007;103:1989-2003. 


\section{Supplementary figure}
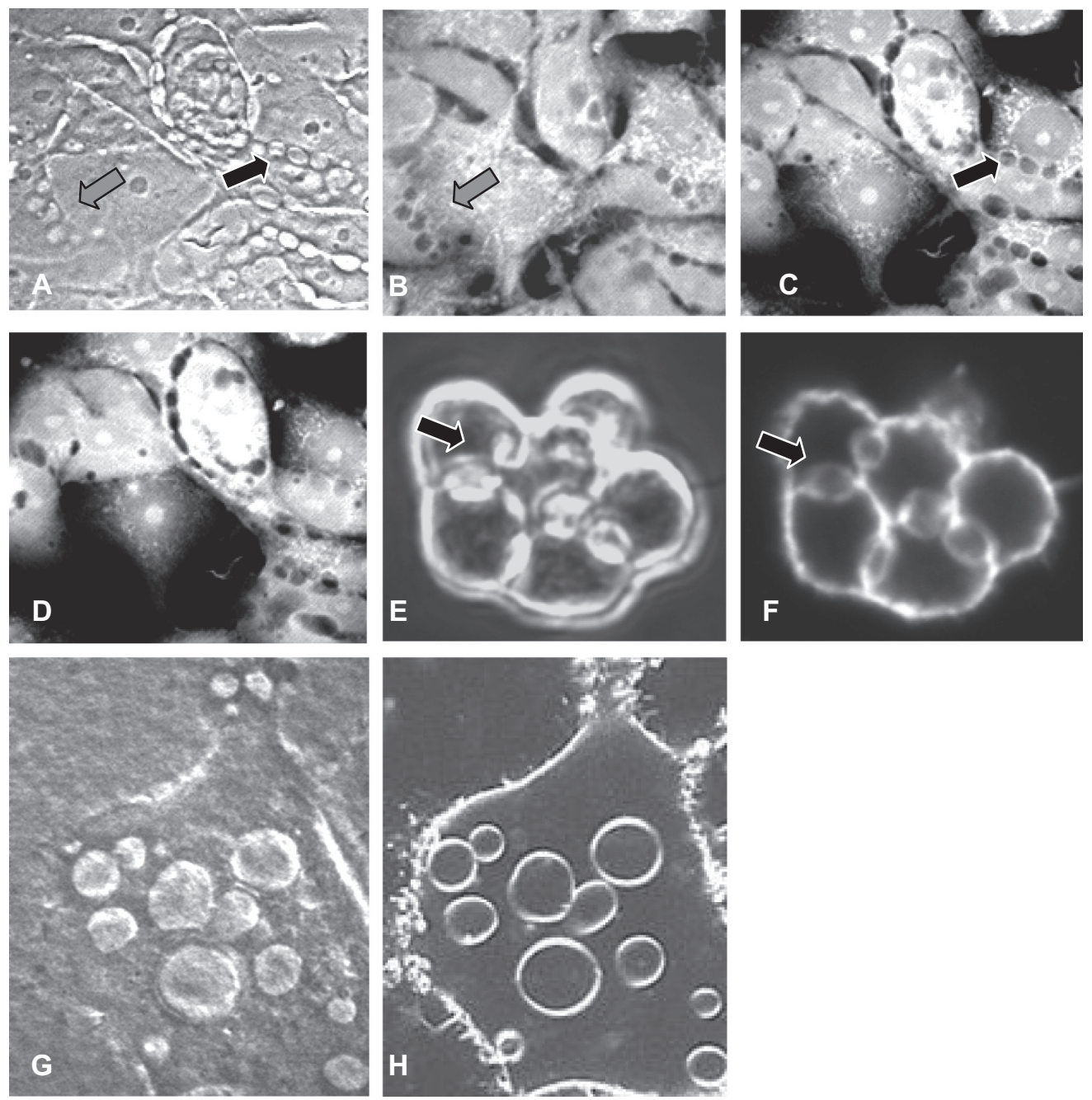

Figure I VLDs in differentiated PCI2 cells (see text). A-D show cells previously loaded with the calcium indicator Fluo-3 to label the cytoplasm. These cells were also transfected with GFP targeted to mitochondria (mtGFP), ${ }^{24}$ so mitochondria appear as intensely fluorescent punctuate structures. A is a phase image (non-confocal) showing multiple VLDs. B-D show three confocal planes of the same cell. Arrows identify VLDs forming in cells and at adherent surfaces between cells. Not all VLDs seen in the phase image are visible in all fluorescence images. $\mathbf{E}$ and $\mathbf{F}$ show VLDs formed at adherent surfaces between $\mathrm{PCI} 2$ cells in suspension; the plasma membrane was labeled with Dil. $\mathbf{E}$ is a phase image and $\mathbf{F}$ is a fluorescence image. $\mathbf{G}$ and $\mathbf{H}$ show a phase image and a simultaneous fluorescence image of a PCI 2 cell labelled with Dil. Since VLDs form from the plasma membrane, the VLD membranes (but not their contents) are labelled by Dil. In the confocal plane shown in $\mathbf{H}$, VLD membranes appear as fluorescent rings, as described elsewhere. ${ }^{-3}$

\section{Publish your work in this journal}

Cell Health and Cytoskeleton is an international, peer-reviewed open access journal focusing on all aspects of cell structure and function contributing to normal physiology and cell health and exploring the pathogenesis of cell dysfunction leading to adverse conditions and disease in the organism. The journal welcomes papers covering original research,

\section{Dovepress}

basic science, reviews and evaluations, guidelines, expert opinion and commentary, case reports and extended reports. The manuscript management system is completely online and includes a very quick and fair peerreview system, which is all easy to use. Visit http://www.dovepress.com/ testimonials.php to read real quotes from published authors. 